\title{
De novo truncating variants in PHF21A cause intellectual disability and craniofacial anomalies
}

\author{
Kohei Hamanaka ${ }^{1}$ Yuji Sugawara ${ }^{2}$ Takeyoshi Shimoji ${ }^{3}$ Tone Irene Nordtveit ${ }^{4}$ - Mitsuhiro Kato ${ }^{5}$ \\ Mitsuko Nakashima ${ }^{6}$. Hirotomo Saitsu $\mathbb{1}^{6}{ }^{6}$. Toshimitsu Suzuki ${ }^{7} \cdot$ Kazuhiro Yamakawa $^{7} \cdot$ Ingvild Aukrust $^{4}$. \\ Gunnar Houge $^{4}$ - Satomi Mitsuhashi ${ }^{1}$ Atsushi Takata ${ }^{1} \cdot$ Kazuhiro Iwama $^{1} \cdot$ Ahmed Alkanaq $^{1} \cdot$ Atsushi Fujita $^{1}$. \\ Eri Imagawa ${ }^{1} \cdot$ Takeshi Mizuguchi $^{1} \cdot$ Noriko Miyake $^{1} \cdot$ Satoko Miyatake $^{1,8} \cdot$ Naomichi Matsumoto $^{1}$
}

Received: 24 May 2018 / Revised: 26 September 2018 / Accepted: 28 September 2018 / Published online: 28 November 2018

(c) European Society of Human Genetics 2018

\begin{abstract}
Potocki-Shaffer syndrome (PSS) is a contiguous gene syndrome caused by $11 \mathrm{p} 11.2$ deletions. PSS is clinically characterized by intellectual disability, craniofacial anomalies, enlarged parietal foramina, and multiple exostoses. PSS occasionally shows autism spectrum disorder, epilepsy, and overgrowth. Some of the clinical features are thought to be associated with haploinsufficiency of two genes in the 11p11.2 region; variants affecting the function of ALX4 cause enlarged parietal foramina and EXT2 lead to multiple exostoses. However, the remaining clinical features were still yet to be linked to specific genetic alterations. In this study, we identified de novo truncating variants in an $11 \mathrm{p} 11.2$ gene, PHF21A, in three cases with intellectual disability and craniofacial anomalies. Among these three cases, autism spectrum disorder was recognized in one case, epilepsy in one case, and overgrowth in two cases. This study shows that PHF21A haploinsufficiency results in intellectual disability and craniofacial anomalies and possibly contributes to susceptibility to autism spectrum disorder, epilepsy, and overgrowth, all of which are PSS features.
\end{abstract}

Naomichi Matsumoto

naomat@yokohama-cu.ac.jp

1 Department of Human Genetics, Yokohama City University Graduate School of Medicine, Yokohama, Kanagawa 236-0004, Japan

2 Department of Pediatrics, Soka Municipal Hospital, Soka, Saitama 340-8560, Japan

3 Department of Neurosurgery, Okinawa Pref. Nanbu Medical Center and Children's Medical Center, Arakawa Haebaru, Okinawa 901-1193, Japan

4 Department of Medical Genetics, Haukeland University Hospital, Bergen 5021, Norway

5 Department of Pediatrics, Showa University School of Medicine, Tokyo 142-8666, Japan

6 Department of Biochemistry, Hamamatsu University School of Medicine, Hamamatsu, Shizuoka 431-3192, Japan

7 Laboratory for Neurogenetics, RIKEN Center for Brain Science, Wako, Saitama 351-0198, Japan

8 Clinical Genetics Department, Yokohama City University Hospital, Yokohama, Kanagawa 236-0004, Japan

\section{Introduction}

Potocki-Shaffer syndrome (PSS) (MIM 601224) is a contiguous gene syndrome caused by 11 p11.2 deletions. PSS is clinically characterized by intellectual disability (ID), craniofacial anomalies (CFA), parietal foramina, and multiple exostoses $[1,2]$. PSS occasionally displays autism spectrum disorders (ASD) [3], epilepsy [3], overgrowth (increased weight, length, and/or head circumference [HC]) [4, 5], and micropenis [4, 6]. The minimum deleted region contains at least five genes [4]. Some clinical features in PSS can be explained by variants affecting function in two genes; in ALX4 (MIM 605420) causing enlarged parietal foramina, and in EXT2 (MIM 608210) causing multiple exostoses [7, 8]. For ID and CFA, PHF21A has been proposed as a good candidate where its variants may cause these features, because three translocations disrupting PHF21A showed ID and CFA (Fig. 1a) [9]. Other PSS clinical features such as ASD, epilepsy, overgrowth, and micropenis remain genetically unexplained. In this study, we describe three cases presenting with ID and CFA arising from de novo truncating variants in the 11p11.2 gene PHF21A (MIM 608325). These cases variably showed other PSS features: ASD, epilepsy, and overgrowth. This study indicates that haploinsufficiency of PHF21A may explain many features of PSS. 

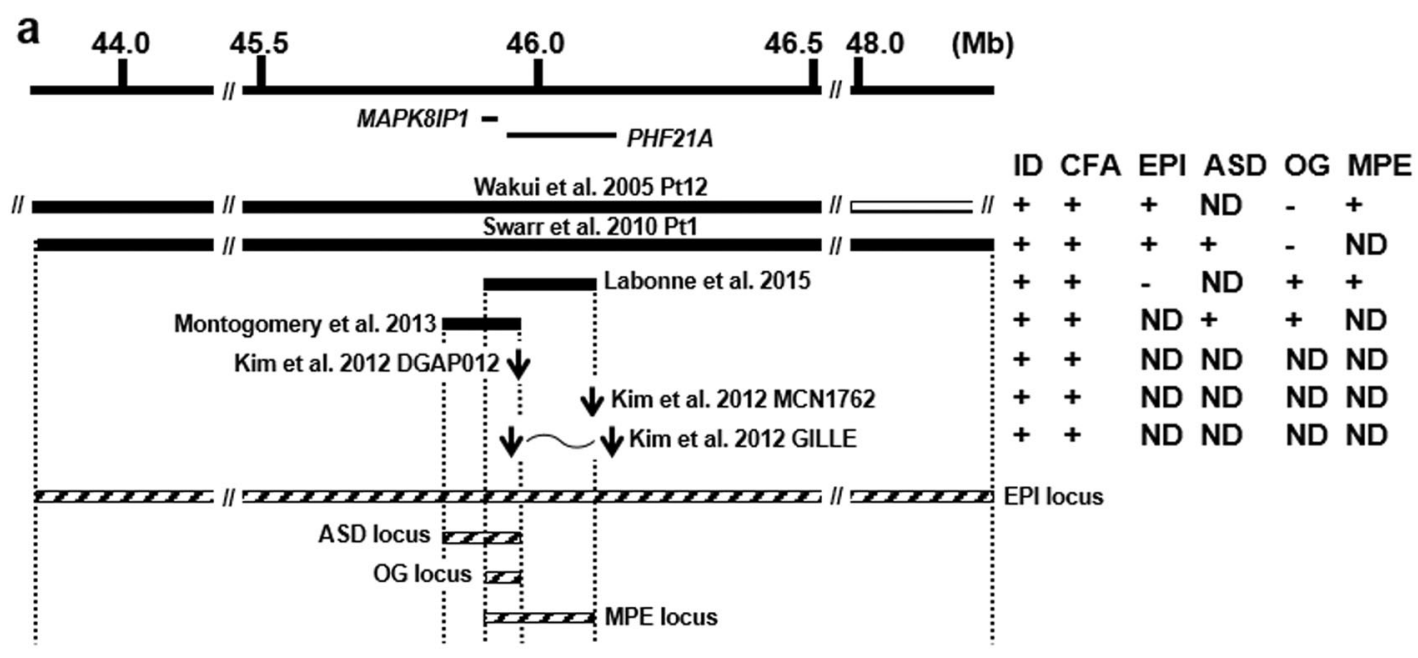

b

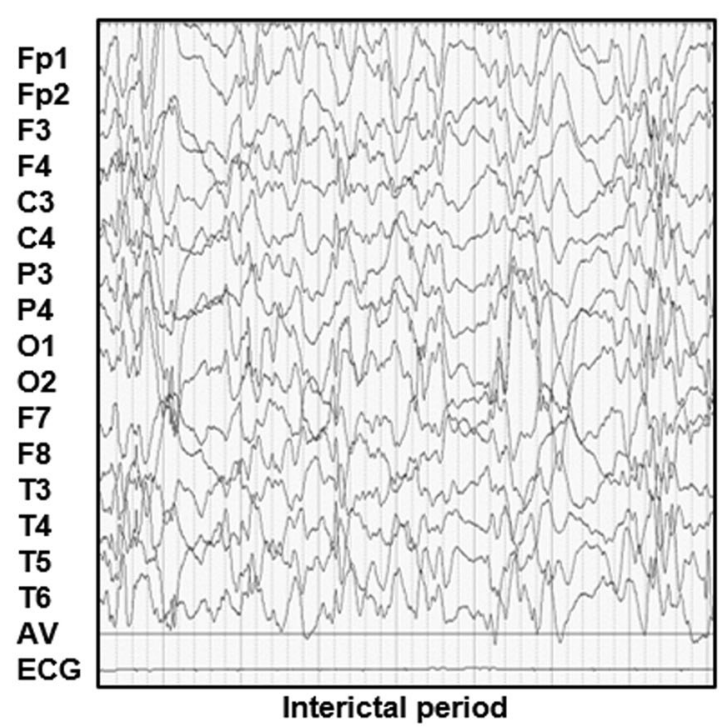

C
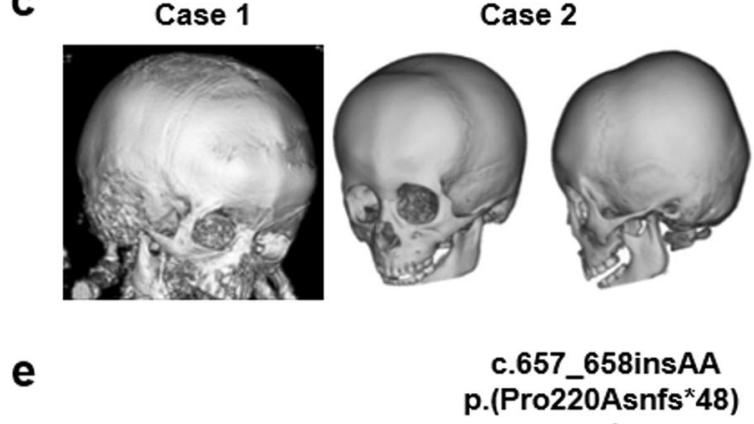

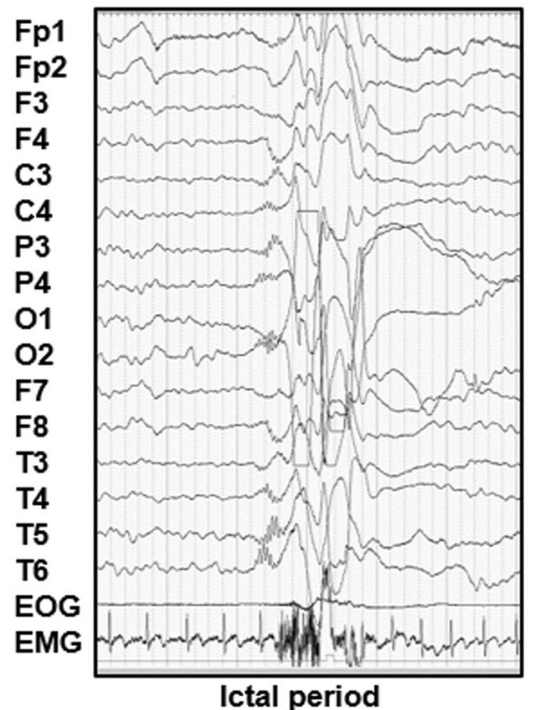

d
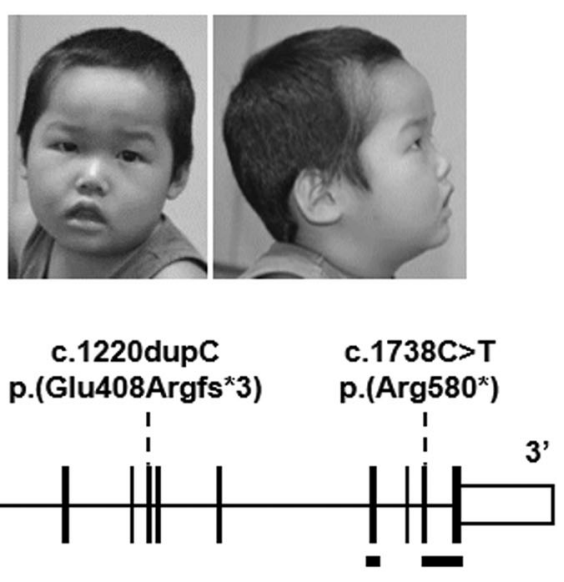

PHD CC 
Fig. 1 Clinical and genetic findings. a Schematic presentation of previously reported deletions and translocations at 11p11-p12 [3-6, 9, $18,19]$. The clinical features observed with each deletion and translocation are shown on the right. Black rectangle: deletion or gene; white rectangle: regions where the breakpoint was not determined; double slash: skipped sequence; down arrow: breakpoints of translocations. The breakpoint of the translocation of the GILLE case was not determined and the range of the breakpoint is shown by down arrows and a wave [9, 18, 19]. Common deleted regions (loci) linked to certain clinical features are shown as patterned rectangles. Genomic chromosome 11 coordinates are shown above the deletions. Mb mega base, ASD ASD-like behavior, EPI epilepsy, OG overgrowth, MPE micropenis, + the symptom was present, - the symptom was absent, ND not determined. b Electroencephalogram of Case 1. Left: nonsynchronous and high spikes and slow waves (hypsarrhythmia) were noted in the interictal period. Right: superimposed high voltage slow waves were noted in the ictal period. ECG electrocardiography, EMG electromyography, EOG electrooculography. c Three-dimensional CT. Left: an image obtained from Case 1 at 18 months, middle and right: images obtained from Case 2 at 5 years. d Facial photographs of Case 1 at 3 years. Brachycephaly, frontal bossing, depressed nasal bridge, small nose with anteverted nares, and downturned mouth with tentshaped upper lip were noted. e Structure of the PHF21A gene. Vertical and horizontal lines indicate exons and introns, respectively. Vertical filled boxes: protein-coding regions; unfilled boxes: untranslated regions. PHF21A variants identified in this study are indicated above PHF21A. Protein domains are indicated below: CC coiled-coil domain, PHD plant homeodomain finger domain

\section{Materials and methods}

\section{Patients}

Informed consent was obtained from the parents of each patient. Additional informed consent was obtained from the parent of Case 1, whose face photographs were included in this article. Genetic analyses of Case 1 and Case 2 were approved by the Institutional Review Board of Yokohama City University School of Medicine (Yokohama, Japan) while Case 3 was diagnosed by routine exome sequencing in Bergen, Norway, which is done without pre-test Institutional Review Board approval. Clinical information was obtained from corresponding clinicians. All procedures performed in this study were in accordance with the Declaration of Helsinki.

\section{Whole-exome sequencing (WES)}

WES for the trios of Case 1 and Case 2 was performed as previously described [10]. In brief, genomic DNA was captured with a SureSelect Human All Exon V5 Kit (Santa Clara, CA, USA, Agilent Technologies) and sequenced on an Illumina HiSeq 2500 (San Diego, CA, USA, Illumina) with 101-bp paired-end reads. Reads were aligned to the human reference genome (GRCh37.1/hg19) using Novoalign v3.02.13 (http://www.novocraft.com/). PCR duplicates were removed using Picard (http://broadinstitute.github.io/ picard/). Local realignments around indels and base quality score recalibration were performed with the Genome Analysis Toolkit (GATK) 3.7-0 (http://www.broadinstitute.org/ gatk/). Variants were called by GATK UnifiedGenotyper and filtered according to GATK Best Practices. Common variants registered in dbSNP135 (minor allele frequency > 0.01 ) that were not flagged as having clinical associations were excluded. Included variants were annotated using ANNOVAR (2016nov) (http://www.openbioinformatics. org/annovar/). WES for the trio of Case 3 was performed as follows. DNA samples were prepared using the SeqCap EZ HGSC VCRome (Roche NimbleGen, Madison, WI) and followed by paired-end $150 \mathrm{nt}$ sequencing on the Illumina NextSeq500. Alignment and variant calling was performed as previously described [11]. Data annotation and interpretation were performed using the Cartagenia Bench Lab, NGS module (Cartagenia, Leuven, Belgium).

\section{Sanger sequencing}

PHF21A variants were analyzed by Sanger sequencing using standard methods. PCR products were sequenced with BigDye Terminator v3.1 Cycle Sequencing kit (Foster City, CA, USA, Applied Biosystems) on a 3500 DNA Sequencing Analyzer (Carlsbad, CA, USA, Life Technologies). Primer sequences are available on request.

\section{Data sharing}

The phenotype and PHF21A variant of cases described in this study were deposited to the gene variant database of Leiden Open Variation Database (https://databases.lovd.nl/ shared/genes/PHF21A). individual IDs were \#00174827, \#00174828, and \#00174829 for Cases 1, 2, and 3, respectively.

\section{Results}

\section{Clinical features}

Case 1 was a 3-year-old boy (Table 1). After an uneventful pregnancy, he was born at term with a birth weight of 4040 $\mathrm{g}$ ( $>97$ th percentile), length of $52 \mathrm{~cm}$ (90-97th percentile), and head circumference of $36.5 \mathrm{~cm}$ ( $>97$ th percentile). He started to experience spasms at the age of 7 months. Developmental milestones were delayed: sitting at 11 months and crawling and speaking incomprehensible words at 18 months. His first clinical evaluation was at 18 months, when electroencephalography revealed hypsarrhythmia in interictal periods and superimposed high voltage slow waves in ictal periods, leading to a diagnosis of West syndrome (Fig. 1b). Computed tomography (CT) was performed at 18 months showing metopic suture closure 
Table 1 Summary of clinical findings

\begin{tabular}{llllll}
\hline Case & PSS $^{\mathrm{a}}$ & MCN1762 & Case 1 & Case 2 & Case 3 \\
\hline Intellectual disability & + & + & + & + & + \\
Speech delay & + & + & + & + & + \\
Motor development delay & + & - & + & + & + \\
Hypotonia & + & + & + & ND & + \\
Epilepsy & + & ND & + & ND & - \\
Brachycephaly & + & + & + & - & - \\
Microcephaly & + & + & Macrocephaly & - & Macrocephaly \\
Metopic ridge & - & ND & + & + & - \\
Prominent forehead & + & ND & + & ND & - \\
Broad nasal tip & + & ND & - & ND & - \\
Depressed nasal tip & + & ND & + & ND & - \\
Narrow nasal bridge & Prominent & + & - & ND & - \\
Short philtrum & + & ND & - & ND & - \\
Downturned corners of mouth & + & + & + & - & + \\
Micrognathia & + & ND & - & ND & - \\
Tapering fingers & + & - & + & - & - \\
Multiple osteochondromas & + & - & - & - & - \\
Enlarged Parietal foramina & + & - & - & ND & - \\
Micropenis & + & Female & - & - \\
\hline+ the symptom was present, & & & + & & - \\
\hline
\end{tabular}

+ the symptom was present, - the symptom was absent, $N D$ not determined

${ }^{a}$ Clinical features previously reported in PSS [2, 3, 6]

${ }^{\mathrm{b}} \mathrm{MCN} 1762$ : a case with translocation disrupting only PHF21A [9] and a metopic ridge but no parietal foramina or exostoses (Fig. 1c, left). His seizures were controlled with adrenocorticotropic hormone therapy followed by the addition of sodium valproate. At 18 months, his weight and height were $13.7 \mathrm{~kg}$ ( $>97 \mathrm{th}$ percentile) and $85 \mathrm{~cm}$ (90-97th percentile), respectively. At 3 years of age, he was not able to understand any speech and had very limited ability to communicate. He was not able to learn any simple daily routine and required close supervision and family care in his daily life. He was diagnosed with severe intellectual disability by DSM-5 criteria. Abnormal craniofacial features were recognized as brachycephaly, frontal bossing, depressed nasal bridge, small nose with anteverted nares, downturned mouth with tent-shaped upper lip, and tapering fingers (Fig. 1d).

Case 2 was a 5-year-old boy (Table 1). He started to manifest signs of developmental delays after his first year of life. He started to walk independently at the age of 2 years. His first visit to the clinic was at 5 years of age, when the physical examination revealed irritability and hyperactivity. Autistic behavior, including aimless head banging, body shaking, and self-harming behavior were noticed. He did not smile and did not make eye contact. The developmental quotient was measured by the K-form developmental test, a commonly used test in Japan, and both cognitive-adaptive and language-social areas were moderately to severely affected. His head circumference was $53.5 \mathrm{~cm}$ (90-97th percentile). Head CT showed a metopic ridge (Fig. 1c, middle and right).

Case 3 (DECIPHER ID: 328685) was a 91/2-year-old Norwegian boy who was referred to a pediatrician at the age of 2 years because of developmental delay and overgrowth (Table 1). He was born at 39 weeks after a normal pregnancy with birth weight of $3.8 \mathrm{~kg}$ (75-90th percentile), length of 51 $\mathrm{cm}$ ( $\sim 50$ th percentile) and head circumference of $36 \mathrm{~cm}$ (97.5th percentile). The neonatal period was uneventful. His parents were healthy with no remarkable family history. He had mild hypotonia with reduced balance and coordination, and started to walk at age 18 months. Development of fine motor skills was also delayed and he struggles with his hand writing. His endurance is impaired. He is often tired and requires much sleep. However, he swims and plays rugby every day. He receives regular physiotherapy. Language development is markedly delayed especially regarding expressive language and articulation. At home he speaks two languages and he has no difficulties with social interactions with his many friends. At school he has "one-to-one teaching" because of learning disabilities, at most mild ID, and attention deficit. He is delayed in performing daily tasks such as dressing himself. He had overeating tendency since birth, for which, he needed nutritional consultations several times. In WWPSI-III (Wechsler Preschool and Primary Scale of Intelligence), a composite score showing the child's general intellectual ability was 76.5 , the borderline level, which 
corresponds to severe learning disability. As expected, his performance score was higher than his verbal score, as his language is severely delayed, which was also found by the PPVT-4 (Peabody Picture Vocabulary Test) for expressive language skills. He was unable to perform the NEPSY-II (Developmental NEuroPSYchological Assessment) for cognitive function, and on the Vineland test communication and motor skills had the lowest scores, while daily living and socialization had scores around at the mean for his age. On the BRIEF (Behavior Rating Inventory of Executive Function) test, a parent and kindergarten report questionaire, the working memory was low. Taken together, his cognitive skills appear to be classified to the severe learning disability/ mild ID range. At age 8 years his weight was $47.5 \mathrm{~kg}(>97.5 \mathrm{th}$ percentile), his height was $143 \mathrm{~cm}$ (97.5th percentile), and his head circumference was $57.2 \mathrm{~cm}$ (97.5th percentile). The skeletal age at chronological age 2 years and 8 months was advanced, corresponding to 3 years and 6 months. He is mildly dysmorphic with downslanting palpebral fissures, mild hypertelorism, a slightly broad nasal bridge, a smooth but not short philtrum, and downturned corners of the mouth. Cerebral MRI performed at 4 years was normal. There are no CT scans of the cranial bones. Hearing and vision is normal. Due to overgrowth, mild ID, and advanced skeletal age, he was initially suspected of having Weaver syndrome.

\section{Variant screening}

Trio-based WES was performed. We identified de novo heterozygous variants in PHF21A (NM_001101802.1): c.1220dupC, p.(Glu408Argfs*3) in Case 1, c.1738C $>$ T, p. $(\operatorname{Arg} 580 *)$ in Case 2, and c.657_658insAA, p. (Pro220Asnfs*48) in Case 3. These PHF21A variants were confirmed by Sanger sequencing and have not been reported as causative variants in the Human Gene Mutation Database (http://www.hgmd.cf.ac.uk/ac/index.php) and are not in public human genome variation databases, such as the Human Genetic Variation Database (http://www.hgvd. genome.med.kyoto-u.ac.jp/), the Exome Variant Server (http://evs.gs.washington.edu/EVS/), the Exome Aggregate Consortium (http://exac.broadinstitute.org/), and the Tohoku Medical Megabank Organization (http://www. megabank.tohoku.ac.jp/tommo). c.657_658insAA is located in exon 8, c.1220dupC is located in exon 12 , and the c. $1738 \mathrm{C}>\mathrm{T}$ variant is in exon 17 , which is the penultimate exon (Fig. 1e). Exons in NM_001101802.1 were here numbered consecutively from the $5^{\prime}$ side.

\section{Discussion}

Several lines of evidence suggest that haploinsufficiency of PHF21A causes ID and CFA as follows. Gross deletions at
$11 \mathrm{p} 11.2$ encompassing PHF21A are associated with ID and CFA [2-6, 12-16]. Gross deletions at 11p11.2 not encompassing $P H F 21 A$ are not associated with ID nor CFA [17]. Balanced translocations disrupting PHF21A show ID and CFA: $\mathrm{t}(11 ; 19)(\mathrm{p} 11.2 ; \mathrm{p} 13.2)\left(\mathrm{c} .1449+2321 \_1449+2322\right) \mathrm{dn}$, $\mathrm{t}(1 ; 11)(\mathrm{p} 21.1 ; \mathrm{p} 11.2)\left(\mathrm{c} .153+34607 \_\mathrm{c} .153+34608\right) \mathrm{dn}$, and $\mathrm{t}$ $(\mathrm{X} ; 11)(\mathrm{p} 22.2 ; \mathrm{p} 11.2) \mathrm{dn}[9,18,19]$. However, its involvement has not been confirmed because the overlapping regions of the deletions (ID/CFA/overgrowth locus in Fig. 1a) contains five genes, MAPK8IPI (MIM 604641), C11orf94 (no MIM number), PEX16 (MIM 603360), GYLTL1B (MIM 609709), and PHF21A. Furthermore, among the three translocations disrupting PHF21A described above, two translocations also break another gene at the other breakpoint: ELAVL1 (MIM 603466) at 19p13.2 in t $(11 ; 19)(\mathrm{p} 11.2 ; \mathrm{p} 13.2) \quad\left(\mathrm{c} .1449+2321 \_1449+2322\right) \mathrm{dn}$ and ARHGAP6 (MIM 300118) at $\mathrm{Xp} 22.2$ in $\mathrm{t}(\mathrm{X} ; 11)(\mathrm{p} 22.2$; p11.2)(c.153+34607_c.153+34608)dn [4, 9]. Moreover, gross deletions and translocations might have position effects on surrounding genes [6]. In this study, we identified three PHF21A-truncating variants in patients with ID and CFA. The 1220dupC p.(Glu408Argfs*3) and c.657_658insAA p.(Pro220Asnfs*48) variants are likely to be functionally null, while the c.1738C $>\mathrm{T}$ p.(Arg580*) variant located in the penultimate exon might not be subjected to nonsense-mediated decay and could form a truncated protein without the $\mathrm{C}$ terminal coiled-coil domain. PHF21A functions as a subunit of a protein complex [9]; therefore, the truncated PHF21A may not be incorporated into the protein complex, resulting in functional impairment. These cases provide direct evidence supporting PHF21A haploinsufficiency as a cause of ID and CFA.

PSS shows variable clinical features: ASD, epilepsy, overgrowth, and micropenis [3-5, 9]. While associations between a deleted gene and clinical features have not been well defined, the cases presented here, with PHF21A-truncating variants, give insight regarding the PSS features that are attributed to PHF21A haploinsufficiency. For example, we observed ASD in Case 2, overgrowth in Case 1 and Case 3, and West syndrome in Case 1. These observations suggest that PHF21A haploinsufficiency contribute to these features, although it might not be fully penetrant. None of the three cases described here had micropenis. PSS cases with micropenis have a deletion containing a minimum of five genes, namely MAPK8IP1, C11orf94, PEX16, GYLTL1B, and PHF21A [4]. Except for PHF21A, only $M A P K 8 I P 1$ has a high probability of being loss-of-function intolerant $(\mathrm{pLI}=1.00)$ according to ExAC. A murine homolog of MAPK8IP1, Jipl, induces chorionic gonadotropin [20]. Chorionic gonadotropin from the placenta induces development of sexual organs in the embryonic male fetus. Thus, MAPK8IPI haploinsufficiency may be a candidate mechanism for micropenis. 
In conclusion, we identified three cases of de novo truncating variants in $P H F 21 A$, highlighting PHF21A haploinsufficiency as a cause of ID and CFA and possibly contributing to susceptibility to ASD, epilepsy, and overgrowth, all of which are PSS features.

Acknowledgements We thank all the participants for their cooperation in this research. We also thank Ms. K. Takabe, Mr. T. Miyama, Ms. N. Watanabe, Ms. M. Sato, Mr. S. Nakamura, and Ms. S. Sugimoto at the Department of Human Genetics, Yokohama City University Graduate School of Medicine, for their technical assistance. This work was supported by AMED under grant numbers, JP18ek0109280, JP18dm0107090, JP18ek0109301, JP18ek0109348, and JP18kk020500; JSPS KAKENHI Grant Numbers, JP17H01539, JP16H05160, JP16H05357, JP16H06254, JP15K10367, JP17K10080, JP 17K15630, and JP17H06994; the fund for Creation of Innovation Centers for Advanced Interdisciplinary Research Areas Program in the Project for Developing Innovation Systems (N Matsumoto) from the Japanese Science and Technology Agency; grants from the Ministry of Health, Labor and Welfare (N Matsumoto); the Takeda Science Foundation (N Miyake, HS, N. Matsumoto); and Kawano Masanori Memorial Public Interest Incorporated Foundation for Promotion of Pediatrics (S Miyatake). This study makes use of data generated by the DECIPHER community. A full list of centers who contributed to the generation of the data is available from http://decipher.sanger.ac.uk and via email from decipher@sanger.ac.uk.

Funding Funding for the decipher project was provided by the Wellcome Trust. We thank Jeremy Allen, PhD, from Edanz Group (www.edanzediting.com/ac) for editing a draft of this manuscript.

\section{Compliance with ethical standards}

Conflict of interest The authors declare that they have no conflict of interest.

\section{References}

1. Romeike BF, Wuyts W. Proximal chromosome $11 \mathrm{p}$ contiguous gene deletion syndrome phenotype: case report and review of the literature. Clin Neuropathol. 2007;26:1-11.

2. Wuyts W, Waeber G, Meinecke P, Schuler H, Goecke TO, Van $\mathrm{Hul} \mathrm{W}$, et al. Proximal $11 \mathrm{p}$ deletion syndrome (P11pDS): additional evaluation of the clinical and molecular aspects. Eur J Hum Genet. 2004;12:400-6.

3. Swarr DT, Bloom D, Lewis RA, Elenberg E, Friedman EM, Glotzbach C, et al. Potocki-Shaffer syndrome: comprehensive clinical assessment, review of the literature, and proposals for medical management. Am J Med Genet A. 2010;152a:565-72.

4. Labonne JD, Vogt J, Reali L, Kong IK, Layman LC, Kim HG. A microdeletion encompassing PHF21A in an individual with global developmental delay and craniofacial anomalies. Am J Med Genet A. 2015;167a:3011-8.

5. Montgomery ND, Turcott CM, Tepperberg JH, McDonald MT, Aylsworth AS. A $137-\mathrm{kb}$ deletion within the Potocki-Shaffer syndrome interval on chromosome $11 \mathrm{p} 11.2$ associated with developmental delay and hypotonia. Am J Med Genet A. 2013;161a:198-202.

6. Wakui K, Gregato G, Ballif BC, Glotzbach CD, Bailey KA, Kuo $\mathrm{PL}$, et al. Construction of a natural panel of 11p11.2 deletions and further delineation of the critical region involved in PotockiShaffer syndrome. Eur J Hum Genet. 2005;13:528-40.

7. Wu YQ, Badano JL, McCaskill C, Vogel H, Potocki L, Shaffer LG. Haploinsufficiency of ALX4 as a potential cause of parietal foramina in the $11 \mathrm{p} 11.2$ contiguous gene-deletion syndrome. Am J Hum Genet. 2000;67:1327-32.

8. Stickens D, Clines G, Burbee D, Ramos P, Thomas S, Hogue D, et al. The EXT2 multiple exostoses gene defines a family of putative tumour suppressor genes. Nat Genet. 1996;14:25-32.

9. Kim HG, Kim HT, Leach NT, Lan F, Ullmann R, Silahtaroglu A, et al. Translocations disrupting PHF21A in the Potocki-Shaffersyndrome region are associated with intellectual disability and craniofacial anomalies. Am J Hum Genet. 2012;91:56-72.

10. Fujita A, Isidor B, Piloquet H, Corre P, Okamoto N, Nakashima $\mathrm{M}$, et al. De novo MEIS2 mutation causes syndromic developmental delay with persistent gastro-esophageal reflux. J Hum Genet. 2016;61:835-8.

11. Bredrup C, Johansson S, Bindoff LA, Sztromwasser P, Krakenes J, Mellgren AE, et al. High myopia-excavated optic disc anomaly associated with a frameshift mutation in the MYC-binding protein 2 gene (MYCBP2). Am J Ophthalmol. 2015;159:973-9.e2.

12. Sohn YB, Yim SY, Cho EH, Kim OH. The first Korean patient with Potocki-Shaffer syndrome: a rare cause of multiple exostoses. J Korean Med Sci. 2015;30:214-7.

13. Romeike BF, Shen Y, Nishimoto HK, Morton CC, Layman LC, Kim HG. Spectrum of genes involved in a unique case of Potocki Schaffer syndrome with a large chromosome 11 deletion. Clin Neuropathol. 2014;33:238-44.

14. Ferrarini A, Gaillard M, Guerry F, Ramelli G, Heidi F, Keddache $\mathrm{CV}$, et al. Potocki-Shaffer deletion encompassing ALX4 in a patient with frontonasal dysplasia phenotype. Am J Med Genet A. 2014;164a:346-52.

15. Palka C, Alfonsi M, Mohn A, Guanciali Franchi P, Chiarelli F, Calabrese G. Delayed diagnosis of Potocki-Shaffer syndrome in a woman with multiple exostoses and mental retardation. Mol Syndromol. 2012;2:259-61.

16. Chuang L, Wakui K, Sue WC, Su MH, Shaffer LG, Kuo PL. Interstitial deletion 11(p11.12p11.2) and analphoid marker formation results in inherited Potocki-Shaffer syndrome. Am J Med Genet A. 2005;133a:180-3.

17. McCool C, Spinks-Franklin A, Noroski LM, Potocki L. PotockiShaffer syndrome in a child without intellectual disability-The role of PHF21A in cognitive function. Am J Med Genet A. 2017;173:716-20.

18. Ansari M, Rainger J, Hanson IM, Williamson KA, Sharkey F, Harewood L, et al. Genetic analysis of 'PAX6-Negative' individuals with Aniridia or Gillespie Syndrome. PLoS ONE. 2016;11: e0153757.

19. Dollfus H, Joanny-Flinois O, Doco-Fenzy M, Veyre L, JoannyFlinois L, Khoury M, et al. Gillespie syndrome phenotype with a t $(\mathrm{X} ; 11)(\mathrm{p} 22.32 ; \mathrm{p} 12)$ de novo translocation. Am J Ophthalmol. 1998;125:397-9.

20. Roberson MS, Ban M, Zhang T, Mulvaney JM. Role of the cyclic AMP response element binding complex and activation of mitogen-activated protein kinases in synergistic activation of the glycoprotein hormone alpha subunit gene by epidermal growth factor and forskolin. Mol Cell Biol. 2000;20:3331-44. 